\title{
THE BRAUER GROUP OF A RING MODULO AN IDEAL
}

\section{F. R. DEMEYER}

Let $R$ be a commutative ring. Let $B(R)$ be the Brauer group of $R$ as defined in [2]. If $I$ is an ideal of $R$ the natural homomorphism from $R$ to $R / I$ induces a homomorphism from $B(R)$ to $B(R / I)$. We study this homomorphism in two contexts. In $\$ 1$ we show that if $I$ is a nilpotent ideal then the homomorphism from $B(R)$ to $B(R / I)$ is an isomorphism. In $\$ 2$ we assume $R$ is a local ring and $I$ is its maximal ideal. We describe the kernel of the homomorphism from $B(R)$ to $B(R / I)$ and then show this homomorphism is onto if $R / I$ is a number field, the $p$-adic completion of a number field, or a function field in one variable over a finite field. The results of $\$ 1$ have been proved by R. Hoobler using cohomological methods, but our proof is algebra theoretic and uses recent results of E. Ingraham [8]. Professor Ingraham provided part of the argument used in the proof of Theorem 1. The results of $\$ 2$ include some of those of V. J. Katz [9] who assumed $R$ to be a regular local domain.

Throughout, our notational conventions will be the same as in [7] which contains the definitions of all unexplained terminology.

Section 1. In this section we prove the following theorem.

THEOREM 1. Let I be a nilpotent ideal in the commutative ring $R$. Then the natural homomorphism from $B(R)$ to $B(R / I)$ is an isomorphism.

Proof. First show the homomorphism is one to one. Let $A$ be a central separable $R$-algebra with $R / I \otimes A$ in the zero class of $B(R / I)$. Then $R / I \otimes A \simeq \operatorname{End}_{R / I}(P)$ for $P$ a finitely generated projective faithful $R / I$-module. We first show there is a finitely generated projective faithful $R$-module $P^{*}$ with $R / I \otimes P^{*}=P$. Let $P_{1}$ be a finitely generated projective $R / I$-module with $P \oplus P_{1}=(I / I)^{n}$. Let $\pi$ be the corresponding projection of $(R / I)^{n}$ onto $P$. Then $\pi$ is an idempotent in $M_{n}(R / I)$, the algebra of $n \times n$ matrices over $R / I$. Moreover, $M_{n}(R / I)=M_{n}(R) / I M_{n}(R)$ and $I M_{n}(R)$ is nilpotent so there is a projection $\pi^{*}$ in $M_{n}(R)$ mapping onto $\pi$ modulo $I \circ M_{n}(R)$. Let $P^{*}=$ $\pi^{*}\left(R^{(n)}\right)$. Then $P^{*}$ is a finitely generated projective $R$-module and the diagram

Received by the editors on July 1, 1974, and in revised form on November 18, 


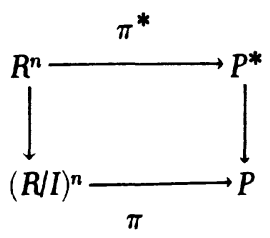

commutes where the vertical arrows are natural. Thus $P^{*} / I P^{*} \simeq P$. By Nakayama's Lemma (Lemma 1.1.7 of [7] $\operatorname{Ann}_{R}\left(P^{*}\right)$ is generated by an idempotent. Since $P$ is faithful, $\operatorname{Ann}_{R}\left(P^{*}\right) \subseteq I$, so $P^{*}$ is faithful by the nilpotence of $I$.

By a theorem of Bass (Corollary 16.2 of [4]) there is a finitely generated projective $R / I$-module $Q$ with $P \otimes Q$ free over $R / I$. With $Q^{*}$ constructed as $P^{*}$ was in the previous paragraph we have

$$
\begin{aligned}
R / I \otimes \operatorname{End}_{R}\left(Q^{*}\right) \otimes A & =(R / I \otimes A) \otimes_{R / I} \operatorname{End}_{R / I}(Q) \\
& =\operatorname{End}_{R / I}(P) \otimes_{R / I} \operatorname{END}_{R / I}(Q) \\
& =\operatorname{End}_{R / I}(P \otimes Q) \\
& =M_{n}(R / I) .
\end{aligned}
$$

We next assert that since $P \otimes Q$ is free over $R / I$, then $P^{*} \otimes Q^{*}$ is free over $R$. Let $F$ be a finitely generated projective $R$-module with $R / I \otimes F$ free over $R / I$. Note $R / I \otimes R=F / I F$ and let $\eta: F \rightarrow F / I F$ be the natural map. Then $\eta$ induces the homomorphism $\phi: \operatorname{End}_{R}(F)$ $\rightarrow \operatorname{End}_{R / I}(F / I F)=M_{n}(R / I)$. Let $1=\sum_{i=1}^{n} \pi_{i}$, where the $\pi_{i}$ are orthogonal projections in $M_{n}(R / I)$ whose images are isomorphic to $R / I$. Lift $\pi_{i}$ to projections $\pi_{i}{ }^{*}$ in $\operatorname{End}_{R}(F)$ so that $\phi\left(\pi_{i}{ }^{*}\right)=\pi_{i}$. Then $F=\oplus \sum_{i=1}^{n} \pi_{i}^{*}(F)$. Let $P_{i}=\pi_{i}^{*}(F)$. The square

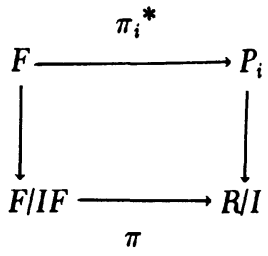

with vertical arrows the natural maps commutes so $P_{i} / I P_{i}=R / I$. This yields the diagram 


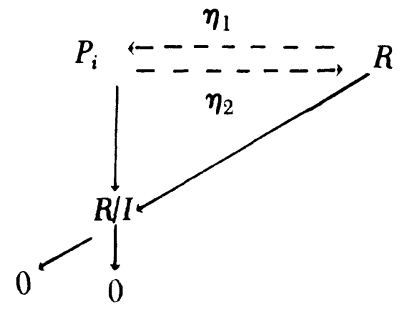

where $\eta_{1}$ and $\eta_{2}$ exist by the projectivity of $R$ and $P_{i}$. now $\eta_{2} \cdot \eta_{1} \equiv 1$ $\bmod I$, so $\eta_{2} \cdot \eta_{1}$ is invertible, $P_{i} \simeq R$ and $F$ is free.

Finally, let $B=A \otimes \operatorname{End}_{R}\left(Q^{*}\right)$. Then $B / I B \simeq M_{n}(R / I)$. Write $1=\sum_{i=1}^{n} \pi_{i}^{*}$ in $B$, where $\pi_{i}^{*}+I B=\pi_{i}$; the $\pi_{i}$ are given in the previous paragraph. Now $\pi_{i} M_{n}(R / I) \pi_{i} \simeq R / I$ so $\pi_{i}{ }^{*} B \pi_{i}{ }^{*}$ is a finitely generated projective $R$-module with homomorphic image $R / I$ by the map

$$
\pi_{i}^{*} B \pi_{i}^{*} \rightarrow \pi_{i}^{*} B \pi_{i}^{*} /\left(\pi_{i}^{*} B \pi_{i}^{*}\right) I .
$$

By the previous paragraph, $\pi_{i}{ }^{*} B \pi_{i}{ }^{*} \simeq R$. Let $\phi: B \rightarrow \operatorname{Hom}_{R}\left(B \pi_{i}{ }^{*}, B \pi_{i}{ }^{*}\right)$ by $\phi(b)\left[x \pi_{i}{ }^{*}\right]=b \times \pi_{i}^{*}$, where we identify $R$ with $\pi_{i}{ }^{*} B \pi_{i}{ }^{*}$. We get the diagram

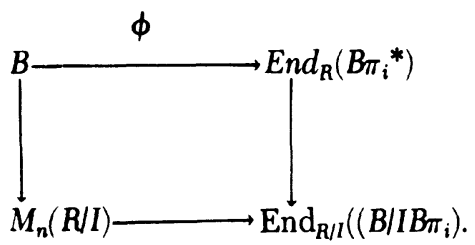

$B_{\pi_{i}}{ }^{*}$ is finitely generated projective over $R$ since $\pi_{i}{ }^{*}$ is idempotent and faithful since $\pi_{i}^{*} B \pi_{i}{ }^{*} \simeq R$. The homomorphism $\phi$ is o e to one on $R$, and so, by Theorem 2.3.7 of [7], is one to one. The image of $\phi$ is a central separable $R$-subalgebra of rank $n^{2}$. Since the bottom arrow is an isomorphism, the rank of $\operatorname{End}_{R}\left(B \pi_{i}{ }^{*}\right)$ is $n^{2}$, so, by Theorem 2.4 .3 of $[7], \dot{\phi}$ is an isomorphism. Thus the homomorphism from $B(R)$ to $B(R / I)$ is one to one.

Next we show the homomorphism from $B(R)$ to $B(R / I)$ is onto. Let $\bar{A}$ be a central separable $R / I$-algebra. By an earlier paragraph there is a finitely generated projective faithful $R$-module $P$ with $P / I P=\bar{A}$ as $R$-modules. We get the diagram 


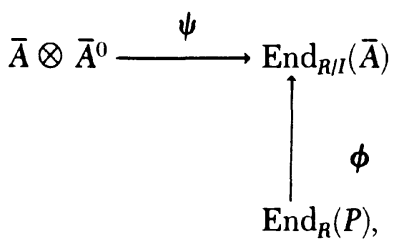

where $\psi(a \otimes b)[x]=a \times b$. Identify $\bar{A}$ with $\psi(\bar{A} \otimes R / I)$ in $\operatorname{End}_{R / I}(\bar{A})$, and let $a_{1}, \cdots, a_{n}$ be elements in $\operatorname{End}_{R}(P)$ mapping by $\phi$ onto the generators of $\bar{A}$. Let $B$ be the $R$-subalgebra of $\operatorname{End}_{R}(P)$ generated by $a_{1}, \cdots, a_{n}$. Then $B / N=\bar{A}$, where $N=B \cap \phi^{-1}(0)$. Let $g_{i} \in N$; then $g_{i} \in \operatorname{Hom}_{R}(P, I P)$. If $n$ is the index of nilpotency of $I$, then

$$
g_{1} \cdots g_{n}(x)=i_{n} \cdots i_{1} y_{n}=0, i_{j} \in I, y_{n} \in P .
$$

Thus $N$ is nilpotent of index $n$. Since $B$ is finitely generated by $a_{1}$, $\cdots, a_{n}$ as an algebra over $R$, and since $B$ is an $R$-subalgebra of $\operatorname{End}_{R}(P)$ so that every $a_{i}$ satisfies a monic polynomial over $R$, we have that $B$ is finitely generated as a module over $R$. By Theorem 1 of [8] there is a separable $R$-subalgebra $A$ of $B$ with $A+N=B$.

We have $A$ is a separable subalgebra of the central separable algebra $\operatorname{End}_{R}(P)$. Thus $\operatorname{End}_{R}(P)$ is finitely generated and projective as a

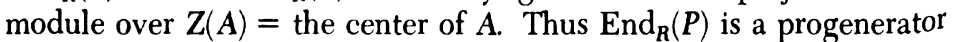
over $Z(A)$, and for some integer $n$ the sequence $\operatorname{End}_{R}(P)^{(n)} \rightarrow Z(A) \rightarrow 0$ is exact and splits over $Z(A)$, and thus over $R$. We conclude that $Z(A)$ is projective over $R$, so $A$ is projective over $R$. But projective separable algebras are finitely generated $(2.2 .1$ of $[1])$, so $A$ is finitely generated over $R$. The ideal $\left.I A \subseteq \operatorname{ker} \phi\right|_{A}$, so $\phi$ induces a homomorphism $\phi: A / I A$ $\rightarrow \bar{A}$. Under $\phi$ the center $Z / I Z$ of $A / I A$ is sent to $R / I$ since $\bar{A}$ is central separable over $R / I$. Thus $Z / I Z \cong R / I \oplus Z_{1} / I Z_{1}$. Since $I$ is nilpotent, idempotents can be lifted modulo $I Z$ so $Z \cong R \oplus Z_{1}$, where $Z_{1} \subseteq$ $\left.\operatorname{ker} \phi\right|_{Z}$. This decomposition of $Z$ yields a corresponding decomposition $A=A_{1} \oplus A_{2}$, where $A_{1}$ is central separable over $R$ and $\left.A_{2} \subseteq \operatorname{ker} \phi\right|_{A}$. Thus $\phi\left(A_{1}\right)=R / I \otimes A_{1} \cong A$, which shows the map from $B(R)$ to $B(R / I)$ is onto.

Section 2. In this section $R$ is a local ring with maximal ideal $m$ and residue class field $k=R / m$. Let $\Omega$ be a separable closure of $R$ (see p. 99 of [7]), and let $S$ be the collection of those finite separable projective extensions $S$ of $R$ in $\Omega$ so that $S / m S=\oplus \sum R / m$. Let $S_{1}, S_{2}$ $\in S$, and let $S_{1} \cdot S_{2}$ be the composite of $S_{1}$ and $S_{2}$ in $\Omega$. Then $S_{1} \cdot S_{2} / m S_{1}$ - $S_{2}$ is an $R$-homomorphic image of

$$
R / m \otimes\left(S_{1} \otimes S_{2}\right)=\oplus \sum R / m .
$$


Thus $S_{1} \cdot S_{2} \in \mathbf{S}$. This demonstrates that $\mathbf{S}$ is a directed set under inclusion. Let $N=\lim \{S \in S\}$, then $N$ is a locally separable extension of $R$ in $\Omega$. If $\sigma \in \operatorname{Aut}_{R}(\Omega)$, then $\sigma(N) \subseteq N$ since $\sigma(S) \in S$ for each $S \in S$. Thus $N$ is a normal extension of $R$ in $\Omega$ called the maximal completely split extension of $R$ in $\Omega$.

THEOREM 2. Let $R$ be a local ring with infinite residue class field $R / m$, and let $N$ be the maximal completely split extension of $R$. Then the sequence

$$
0 \rightarrow B(N / R) \rightarrow B(R) \rightarrow B(R / m)
$$

is exact.

Proof. Let $|A| \in B(N / R)$. Then $N \otimes A=\operatorname{Hom}_{N}(P, P)$ for a finitely generated projective $N$-module $P$. The proof of proposition 3 in [6] shows that $P$ is a free $N$-module. Let $x_{1}, \cdots, x_{n}$ be a basis for $A$ over $R$. Each $1 \otimes x_{i}$ is an $n \times n$ martix $A_{i}$ over $N$. Find an element $S \in S$ containing the $n^{3}$ elements of $N$ in these matrices. Then $S \otimes A \simeq$ $M_{n}(S)$ by $1 \otimes x_{i} \rightarrow A_{i} . \quad$ Now $R / m \otimes S \otimes A \simeq\left(\oplus \sum R / m\right) \otimes A \simeq \oplus$ $\sum(R / m \otimes A) \simeq \sum M_{n}(R / m)$, thus $R / m \otimes A$ is in the zero class of $B(R / m)$.

Now let $|A| \in B(R)$ with $R / m \otimes A$ in the zero class of $B(R / m)$. Then $R / m \otimes A \simeq A / m A \simeq M_{n}(R / m)$. Since $R / m$ is infinite we can choose a diagonal matrix $\bar{\theta}$ in $M_{n}(R / m)$ with distinct entries $\beta_{i}$ on the diagonal. Now $\bar{\theta}$ satisfies $\prod_{i=1}^{n}\left(\beta_{i}-x\right)=p(x)$ which is a separable polynomial over $R / m$. Also, $R / m(\bar{\theta})$ is a maximal commutative subalgebra of $M_{n}(R / m)$ since $R / m(\bar{\theta})$ includes all diagonal matrices.

Let $\theta \in A$ with $\theta$ mapping to $\bar{\theta}$ under the mapping from $A$ to $A / m A$. Let $S=R \cdot 1+R \theta+\cdots+R \theta^{n-1}$. Since $\left\{1, \theta, \cdots, \theta^{n-1}\right\}$ is a free basis for an $R / m$-direct summand of $A / m A$, Nakayama's Lemma (pg. 377 of [2]) implies that $\left\{1, \theta, \cdots, \theta^{n-1}\right\}$ is a free set of generators of $S$ over $R$ which extends to a free set of generators of $A$ over $R$, so $S$ is an $R$-module direct summand of $A$.

Now we show $S$ is a subring of $A$ by showing $\theta^{n} \in S$. Let $\bar{R}$ be the Henselization of $R$ and let $\bar{A}=\bar{R} \otimes A$. Then $A / m A \simeq \bar{A} / m \bar{A}$. Since $\bar{R}$ is Henselian and $\bar{A} / m \bar{A}$ is in the zero class of $B(\bar{R} / m \bar{R}), A$ is in the zero class of $B(R)$ as is shown in [3]. Thus $\bar{A} \simeq M_{n}(\bar{R})$, and $A$ is a subring of $\bar{A}$. By the Cayley-Hamilton Theorem, $\theta$ satisfies a monic polynomial of degree $n$ over $R$. But $S$ is an $R$ direct summand of $A$, and so, since $\theta^{n} \in \bar{R} \otimes S$, we have $\theta^{n} \in S$.

$S$ is separable over $R$ since $S \cap m S=S \cap m A$, and thus $S / m S=$ $R / m(\bar{\theta})$, which is separable over $R / m$. Let $S^{*}$ be the commutant of 
$S$ of $A$; by Theorem 4.3 of [7], $S^{*}$ is a finitely generated projective $R$ algebra, and the commutant in $A$ of $S^{*}$ is $S$. But $S^{*} \cap m A$ is a twosided ideal in $S^{*}$, so, by Corollary 3.2 of [2], there is an ideal $M$ of $S$ with $M S^{*}=S^{*} \cap m A$. But $M S^{*} \cap S^{*}=M=\left(S^{*} \cap m A\right) \cap S=m S$, so $M=m S$ and $S^{*}=m S^{*}+S$. By Nakayama's Lemma, $S^{*}=S$, so by Theorem 5.5 of [7], $S \otimes_{R} A$ is in the zero class of $B(S)$. Observe that $S \in S$ since $S / m S=R / m(\bar{\theta})=\oplus \sum R / m$. This proves the theorem.

If $R / m$ is a finite field then $B(R / m)$ is trivial so the natural map from $B(R)$ to $B(R / m)$ is onto and every element of $B(R)$ is in the kernel. Our last theorem gives another condition under which the homomorphism from $B(R)$ to $B(R / m)$ is onto.

TheOREM 3. Let $R$ be a local ring with maximal ideal $m$ and residue class field $k=R / m$. Assume that every element of $B(k)$ of order $p^{m}$ for $p$ a prime is split by an extension $k(\epsilon)$ of $k$ where $\epsilon$ is a $p^{n}$-th root of unity. Then the homomorphism $B(R) \rightarrow B(k)$ is onto.

Proof. Every element in $B(k)$ is a product of elements of prime power order. Thus it suffices to show that each such element is in the image of the map from $B(R)$ to $B(k)$. Let $|A|$ be a class in $B(k)$ of order $p^{m}, p$ a prime. Then $A \sim \Delta(k(\boldsymbol{\epsilon}), H, \boldsymbol{\beta})$, where $\epsilon$ is a primitive $p^{n}$-th root of $1, H=\mathrm{Gal}(k(\epsilon) / k)$, and $\beta \in Z^{2}\left(H, k(\epsilon)^{*}\right)$.

Case 1. $p$ is odd.

In this case $H$ is cyclic. Let $S=R(\epsilon)$, where $\epsilon$ is a primitive $p^{n}$-th root of 1 over $R$. Then $S$ is a Galois extension of $R$ with cyclic Galois group $G$. Moreover, $S / m S \simeq k(\epsilon) \oplus \cdots \oplus k(\epsilon)$ is a Galois extension of $k$ with group $G$. Note $S / m S$ splits $|A|$, so $|A|=|\Delta(S / m S, G, \rho)|$, where if $G=\langle\sigma\rangle$, then one can choose $\rho$ so that

$$
\rho\left(\boldsymbol{\sigma}^{i}, \boldsymbol{\sigma}^{j}\right)=\left\{\begin{array}{l}
1, i+j<o(\langle\boldsymbol{\sigma}\rangle) \\
\bar{a}, i+j \geqq o(\langle\boldsymbol{\sigma}\rangle), \bar{a} \in k .
\end{array}\right.
$$

Let $|\Delta(S, G, \alpha)|$ be in $B(R)$, where

$$
\boldsymbol{\alpha}\left(\boldsymbol{\sigma}^{i}, \boldsymbol{\sigma}^{j}\right)=\left\{\begin{array}{l}
1, i+j<o(\langle\boldsymbol{\sigma}\rangle) \\
a, i+j \geqq o(\langle\boldsymbol{\sigma}\rangle), a \in R, a+m=\bar{a} .
\end{array}\right.
$$

Then clearly, $|\Delta(S, G, \alpha)|$ maps onto $|A|$.

Case 2. $p=2$.

Subcase $1 . \sqrt{-1} \in k$ and $\sqrt{-1} \in R$. In this case choose $\epsilon, S$ as in Case 1, then $H, G$ are cyclic and one proceeds as in Case 1 .

Subcase 2. $\sqrt{-1} \notin k$.

In this case $k(\epsilon)$ and $R(\epsilon)$ have the same rank over $k, R$, respectively, so $R(\epsilon) / m R(\epsilon) \simeq k(\epsilon)$, and $R(\epsilon)$ is a local ring. By proceeding as in the 
proof of Theorem 53.3 of [5], one can, by adjoining roots of polynomials of the form $x^{2^{k}}-\bar{a}$ to $k(\epsilon)$, write a 2 -cocycle $\bar{\beta}$ on an extension $K$ of $k(\epsilon)$ with Galois group $\bar{H}$ over $k$ with the values of $\bar{\beta}$ in roots of unity and $|A|=|\Delta(K, \bar{H}, \bar{\beta})|$. Because $R(\epsilon)$ and $k(\epsilon)$ both have a primitive $2^{n}$-th root of 1 , one can construct by Kummer's Theory ( $\mathrm{p}$. 130 of [7]) an extension $N$ of $R(\epsilon)$ obtained by adjoining roots of polynomials of the form $x^{2^{k}}-a$ to $R(\epsilon)$, where $a+m=\bar{a}, N / m N$ $=K$, and $\operatorname{Gal}(N / R)=\bar{H}$. Consider $\Delta(N, \bar{H}, \beta)$, where $\beta$ has the same root of unity values as $\bar{\beta}$. Clearly $|\Delta(N, \bar{H}, \beta)|$ maps into $|\Delta(k, \bar{H}, \beta)|=|A|$.

Subcase 3. $\sqrt{-1} \in k$, but $\sqrt{-1} \notin R$.

In this case $|A|=\mid \Delta(k(\epsilon), H, a)$ where $H$ is cyclic. Also $R(\epsilon)$ is a normal separable extension of $R$ with Galois group $G=C_{2} \times C_{n}$ (where $C_{n}$ is the cyclic group of order $n$ and $n=2^{k}$ ). Moreover,

$$
\begin{aligned}
R(\boldsymbol{\epsilon}) / m R(\boldsymbol{\epsilon}) & =[k(\boldsymbol{\epsilon}) \oplus \cdots \oplus k(\boldsymbol{\epsilon})] \oplus[k(\boldsymbol{\epsilon}) \oplus \cdots \oplus k(\boldsymbol{\epsilon})] \\
& =N \oplus N
\end{aligned}
$$

is a Galois extension of $R / m$ with Galois group $G$, where $N$ is identified with either term in square brackets. Write $G=\langle\sigma\rangle \times\langle\tau\rangle$ with $\sigma^{2}=e, \tau^{n}=e$. Then $N \simeq\{(\alpha, \alpha) \mid \alpha \in N\}=(N \otimes N)^{\langle\sigma\rangle}$ is a Galois extension of $k$ with cyclic Galois group $\langle\tau\rangle$, and $A$ is split by $N$. Thus $A=|\Delta(N,\langle\tau\rangle, \bar{\beta})|$, where

$$
\bar{\beta}\left(\tau^{i}, \tau^{j}\right)= \begin{cases}1, & i+j<n \\ \bar{a}, & i+j \geqq n, \quad \bar{a} \in k .\end{cases}
$$

Exactly as in Theorem $8.15 \mathrm{E}$ of $[1]$, we have $A=|\Delta(N \oplus N, G, \beta)|$, where

$$
\boldsymbol{\beta}\left(\boldsymbol{\sigma}^{i} \tau^{j}, \boldsymbol{\sigma}^{k} \tau^{l}\right)= \begin{cases}1, & j+l<n \\ \bar{a}, & j+l \geqq n .\end{cases}
$$

Let $|\Delta(R(\boldsymbol{\epsilon}), G, \boldsymbol{\alpha})|$ be an element in $B(R)$ with $\boldsymbol{\alpha}$ given by

$$
\alpha\left(\boldsymbol{\sigma}^{i} \tau^{j}, \boldsymbol{\sigma}^{k} \tau^{l}\right)= \begin{cases}1, & j+l<n \\ a, & j+l \geqq n, a \in R, a+m=\bar{a} .\end{cases}
$$

Again, $|A|$ is the image of $|\Delta(R(\epsilon), G, \alpha)|$. This completes the proof.

The hypothesis of Theorem 3 is satisfied by algebraic number fields, function fields in one variable over finite fields, and $p$-adic completions of number fields. We thus have the following theorem. 
THEOREM 4. Let $R$ be a local ring with maximal ideal $m$ and residue class field $R / m=k$. Let $N$ be the maximal completely split extension of $R$. If $k$ is an algebraic number field, a function field in one variable over a finite field, or the p-adic completion of a number field, then the sequence

$$
0 \rightarrow B(N / R) \rightarrow B(R) \rightarrow B(R / m) \rightarrow 0
$$

is exact.

\section{BiBLIOGRAPHY}

1. M. Artin, C. Nesbitt, and R. M. Thrall, Rings with Minimum Condition, U. of Michigan Press, Ann Arbor 1944.

2. M. Auslander and O. Goldman, The Brauer Group of a Commutative Ring, Trans. Amer. Math. Soc. 97 (1960), 367-409, MR 22 \# 12130.

3. G. Azumaya, On Maximally Central Algebras, Nagoya Math. J. 2 (1951), $119-150$.

4. H. Bass, K Theory and Stable Algebra, Publ. I.H.E.S. \#22 (1964), 5-60.

5. C. W. Curtis and I. Reiner, Representation Theory of Finite Groups and Associative Algebras, Interscience (1962).

6. F. DeMeyer, The Brauer Group of Some Separably Closed Rings, Osaka Math. J. 3 (1966), 201-204, MR 35 \#2881.

7. F. DeMeyer and E. Ingraham, Separable Algebras over Commutative Rings, Springer Verlag, Lecture Notes in Mathematics \#181 (1971).

8. E. Ingraham, On the Existence and Conjugacy of Inertial Subalgebras, Journal of Algebra 31 (1974), 547-556.

9. V. J. Katz, The Brauer Group of a Regular Local Ring, Ph.D. Thesis, Brandeis U. (1968).

10. D. Sanders, The Dominion and Separable Subalgebras of Finitely Generated Algebras, Proceedings Amer. Math. Soc. 48 (1975), 1-7.

Colorado State University, Ft. Collins, Colorado 80521 